\title{
Unsteady magnetohydrodynamic flow of some non-Newtonian fluids with slip through porous channel
}

\author{
Funmilayo H. Oyelami ${ }^{1,2 *}$, Moses S. Dada ${ }^{2}$ \\ ${ }^{1}$ Department of Mathematical and physical sciences, Afe Babalola University, Ado Ekiti 360001, Nigeria \\ ${ }^{2}$ Department of Mathematics, University of Ilorin, Ilorin 240101, Nigeria
}

Corresponding Author Email: adefolajufunmilayo@gmail.com

https://doi.org/10.18280/ijht.360237

Received: 20 December 2017

Accepted: 3 April 2018

\section{Keywords:}

non-Newtonian fluid, slip, porous medium, eyring-powell model, prandtleyring model

\begin{abstract}
The numerical analysis for transfer of heat by natural convection on an unsteady Magnetohydrodynamic flow of non-Newtonian fluids through porous channel is considered. Equations governing the model are formulated, simplified and non-dimensionalised. The solution is obtained by employing Crank Nicolson's type of finite difference discritization. Velocity as well as the temperature distributions for both Prandtl-Eyring and Eyring-Powell non-Newtonian fluid models are examined. Comparism between these two diverse liquid models is made with their graphical illustrations on velocity and temperature profiles. It is observed that the velocity is higher for Prandtl Eyring model than Eyring Powell model. Also, the temperature variation for Prandtl number in Eyring-Powell fluid is a little slower than that of Prandtl-Eyring fluid.
\end{abstract}

\section{INTRODUCTION}

Fluid can be regarded as a material in nature that deforms continually under applied shear stress. Non-Newtonian fluids are fluids that do not obey the Newtonian relationship between the shear stress and shear rate. High molecular weight liquids, which include polymer melts and solutions of polymers, such as liquids in which fine particles are suspended like slurries, pastes, gels, polymer solutions are usually referred to as nonNewtonian fluids.

These non-Newtonian fluids have non-linear relationship between shear stress and shear strain.

Several models with different rheological features have been established to describe the non-linear relationship between shear stress and shear strain. Examples of these models are Power-law model, Williamson model, EyringPowell model, Prandtl-Eyring model, Prandtl model, and so on

Some researchers distant themselves from the study of nonNewtonian fluids because of the complexity of these models. The few that find it easy prefer to use Power-law model because of its simple rheological features over a limited shearing rate. An advantage of Eyring-Powell model over Power-law model is that it accurately lessens to Newtonian behaviour for low and high shear rate.

Also, Prandtl-Eyring model is of great importance in the manufacturing industries in the formulation of flow for ethylene glycol and powdered graphite.

Timol and Kalthia [1] worked on the natural convection flows of all kinds of non-Newtonian visco inelastic fluids using systematic similarity analysis. Eldabe et al. [2] studied the one directional flow of non-Newtonian Powell-Eyring fluid with coupled stresses through parallel plates. Zueco and Beg [3] considered a pulsatile flow of Powell-Eyring fluid.

Asmat et al. [4] studied radiation effects on boundary layer flow of a non-Newtonian Eyring-Powell fluid over a sheet that is exponentially shrinking.
Using an Eyring-Powell fluid model, Malik et al. [5] studied boundary layer flow due to a stretching cylinder with variable viscosity. On the radiation effects affecting the flow of PowellEyring fluid, Tasawar et al. [6] examined the non-Newtonian fluid past an unsteady inclined stretching sheet having nonuniform heat source and sink.

Also, numerical studies was carried out by Khader and Megahed [7] to study the heat transfer from an unsteady Powell-Eyring fluid with a stretching sheet over a thin film.

Arifuzzaman et al. [8] investigated a Chemically reactive viscoelastic fluid flowing in presence of nano particle through porous stretching sheet. Khan et al. [9] studied thermal radiation and viscous dissipation effects on an unsteady magnetohydrodynamic free convection boundary-layer flow of a nanofluid along a stretching sheet. Cross Diffusion and magnetohydrodynamic effect on a high order chemically reactive micropolar fluid of naturally convective heat and mass transfer through an Infinite vertical porous medium with a Constant Heat Sink was considered by Arifuzzaman et al. [10]. MHD Maxwell fluid flow in presence of nano-particle through a vertical porous-plate with heat- generation, radiation absorption and chemical reaction was examined by Arifuzzaman et al. [11].

Zaman et al. [12] investigated an unsteady incompressible couette flow of Powell-Eyring non-Newtonian fluid under a uniform suction and injection. For an unsteady MHD NonNewtonian fluid having slip conditions in a porous medium, Gbadeyan and Dada [13] investigated the transfer of heat and radiation in the fluid. Adesanya and Gbadeyan [14] studied visco-elastic fluid flow having slip conditions in a planer medium using adomian decomposition method. Ahmed and Imen [15] examined the effect of variable thermal conductivity/refractive index on an analysis of conductionradiation heat transfer by applying Lattice Boltzmann method.

Motivation of this present work comes from the work done by Darji and Timol [16] where they carried out a research that 
involved the similarity analysis between Williamson and Prandtl-Eyring models for a natural convection boundary layer flow. It was observed in their work that the velocity in Williamson model is higher than that of Prandtl-Eyring model. In this manner, this work aimed at studying and comparing the flow of Prandtl Eyring and Eyring Powell models on an unsteady magnetohydrodynamic flow of non-Newtonian fluid in a porous medium.

\section{PROBLEM FORMULATION}

Unsteady incompressible non-Newtonian fluid through vertical parallel plates of width $h$ filled with porous medium is examined, with its $x^{*}$-axis selected ahead one of the plates and the $y^{*}$-axis is chosen normal to it under the action of a perpendicularly applied magnetic field having a uniform strength. Using Boussinesq approximation for incompressible fluid model, the governing equations in terms of momentum and energy equations are as follows:

$\frac{\partial u^{*}}{\partial t^{*}}=-\frac{1}{\rho} \frac{\partial P^{*}}{\partial x^{*}}-\frac{1}{\rho} \frac{\partial \tau_{x y}}{\partial y^{*}}+\mathrm{g} \beta_{T}\left(T^{*}-T_{0}^{*}\right)-\frac{1}{\rho} \sigma B_{0}^{2} u^{*}-\frac{v u^{*}}{K}$

$\frac{\partial T^{*}}{\partial t^{*}}=\frac{k}{\rho C_{p}} \frac{\partial^{2} T^{*}}{\partial y^{* 2}}-\frac{1}{\rho C_{p}} \frac{\partial q_{r}}{\partial y^{*}}+\frac{v}{C_{p}}\left(\frac{\partial u^{*}}{\partial y^{*}}\right)^{2}$

where $u^{*}$ is the velocity of the fluid, $t^{*}$ is the time, $\rho$ implies fluid density, $g$ stands for acceleration due to gravity, $P^{*}$ implies fluid pressure, $C_{p}$ stands for specific heat at constant pressure, $T^{*}$ implies fluid temperature, $\beta_{T}$ equates to coefficient of thermal expansion, $\mathrm{k}$ represent the thermal conductivity, $T_{w}^{*}$ stands for temperature of the fluid at $y^{*}=h$ while $T_{0}^{*}$ stands for temperature of the fluid at $y^{*}=0, q_{r}$ represents the radiative heat flux, $\sigma$ implies electrical conductivity, $v$ equates to kinematic viscosity, $B_{0}$ represents the magnetic field and $\mathrm{h}$ stands for the distance between two plates.

The underlying and limit conditions are given as

$$
\begin{aligned}
& t^{*}=0 \quad u^{*}=0 \quad T^{*}=T_{0}^{*} \quad y \in(0, h) \\
& t^{*}>0, \quad u^{*}=\lambda \frac{\partial u^{*}}{\partial y^{*}} \quad T^{*}=T_{0}^{*} \quad \text { about } \quad y^{*}=0 \\
& u^{*}=0 \quad T^{*}=T_{w}^{*} \quad \text { about } \quad y^{*}=h
\end{aligned}
$$

Following Gbadeyan and Dada [13], the radiation parameter is given as

$\frac{\partial q_{r}}{\partial y^{*}}=4\left(T^{*}-T_{0}^{*}\right) L$

where $\mathrm{L}=\int_{0}^{\infty} k \lambda_{w}\left(\frac{d e_{b \lambda}}{d T^{*}}\right)_{w} d \lambda, k \lambda_{w}$ represents coefficient of absorption and $e_{b \lambda}$ stands for the plank constant.

By defining the following non-dimensional quantities,

$X=\frac{x^{*}}{h}, Y=\frac{y^{*}}{h}, U=\frac{u^{*}}{u_{0}^{*}}, t=\frac{t^{*} u_{0}^{*}}{h}, P=\frac{P^{*}}{\rho u_{0}^{* 2}}, T=$ $\frac{T^{*}-T_{0}^{*}}{T_{w}^{*}-T_{0}^{*}}, R e=\frac{h u_{0}^{*}}{v}, E c=\frac{u_{0}^{* 2}}{C p\left(T_{w}^{*}-T_{0}^{*}\right)}, N=\frac{4 L h^{2}}{k}, \operatorname{Pr}=\frac{\rho C p v}{k}$, $D a=\frac{h^{2}}{K}, G r=\frac{g \beta_{T}\left(T_{w}^{*}-T_{0}^{*}\right) h^{2}}{v}, M^{2}=\frac{\sigma B_{0}^{2} h^{2}}{\rho v}, E c=\frac{u_{0}^{* 2}}{C_{p}\left(T_{w}^{*}-T_{0}^{*}\right)}$,

Introducing equation (4) into equations (2) under the expression in equations (5), equation (2) becomes
$R e \frac{\partial T}{\partial t}=\frac{1}{P r} \frac{\partial^{2} T}{\partial Y^{2}}-\frac{N}{P r} T+E c\left(\frac{\partial U}{\partial Y}\right)^{2}$

With the relating dimensionless limit conditions

$\begin{array}{lllll}t=0 & U=0 & T=0 & & Y \in(0, h) \\ t>0, & U=\lambda \frac{\partial U}{\partial Y} & T=0 & \text { about } & Y=0 \\ & U=0, & T=1, & \text { about } & Y=1\end{array}$

Model for Prandtl-Eyring non-Newtonian fluid is defined according to Darji and Timol (16) as

$\tau_{x y}=\beta \sinh ^{-1}\left(\frac{1}{b} \frac{\partial u^{*}}{\partial y^{*}}\right)$

with $\tau_{x y}, \frac{\partial u^{*}}{\partial y^{*}}, \beta$ and $b$ representing the shear stress, velocity gradient and Prandtl-Eyring parameters respectively.

From equation (8) above, $\sin h^{-1}\left(\frac{1}{b} \frac{\partial u^{*}}{\partial y^{*}}\right)=\frac{1}{b} \frac{\partial u^{*}}{\partial y^{*}}-$ $\frac{1}{6}\left(\frac{1}{b} \frac{\partial u^{*}}{\partial y^{*}}\right)^{3}+\frac{3}{40}\left(\frac{1}{b} \frac{\partial u^{*}}{\partial y^{*}}\right)^{5}-\frac{5}{112}\left(\frac{1}{b} \frac{\partial u^{*}}{\partial y^{*}}\right)^{7}+\cdots$

Taking the first term in the series expansion above,

$\sin h^{-1}\left(\frac{1}{b} \frac{\partial u^{*}}{\partial y^{*}}\right) \cong \frac{1}{b} \frac{\partial u^{*}}{\partial y^{*}},\left|\frac{1}{b} \frac{\partial u^{*}}{\partial y^{*}}\right| \ll 1$

The stress tensor for Prandtl-Eyring model is given as

$\tau_{x y}=\frac{\beta}{b} \frac{\partial u^{*}}{\partial y^{*}}$

By introducing equations (5) and (10) into equation (1), the momentum equation for Prandtl-Eyring model becomes

$R e \frac{\partial U}{\partial t}=-\frac{\partial P}{\partial X}+\eta \frac{\partial^{2} U}{\partial Y^{2}}+G_{r} T-\left(M^{2}+D a\right) U$

where $\eta=\frac{\beta}{b \mu}$ is the characteristics of Prandtl-Eyring model.

Also, model for Eyring-Powell non-Newtonian fluid is modified according to Adesanya and Gbadeyan [14] as

$T_{x y}=\mu \frac{\partial u^{*}}{\partial y^{*}}+\frac{1}{\alpha} \sinh ^{-1}\left(\frac{1}{c} \frac{\partial u^{*}}{\partial y^{*}}\right)$

with $\tau_{x y}, \frac{\partial u^{*}}{\partial y^{*}}, \mu, \alpha$ and $c$ signifying the shear stress, velocity gradient, coefficient of dynamic viscosity and the EyringPowell fluid parameters respectively.

From equation (12), the hyperbolic sine function of first order is given as

$\sin h^{-1}\left(\frac{1}{c} \frac{\partial u^{*}}{\partial y^{*}}\right) \cong \frac{1}{c} \frac{\partial u^{*}}{\partial y^{*}},\left|\frac{1}{c} \frac{\partial u^{*}}{\partial y^{*}}\right| \ll 1$

Introducing equation (13) into equation (12), stress tensor for Eyring-Powell fluid reduces to

$T_{x y}=\mu \frac{\partial u^{*}}{\partial y^{*}}+\frac{1}{\alpha c}\left[\frac{\partial u^{*}}{\partial y^{*}}\right]$

By introducing equations (5) and (14) into equation (1), the momentum equation for Eyring-Powell model becomes

$R e \frac{\partial U}{\partial t}=-\frac{\partial P}{\partial X}+[1+A] \frac{\partial^{2} U}{\partial Y^{2}}+G_{r} T-\left(M^{2}+D a\right) U$ 
where $\mathrm{A}=\frac{1}{\alpha \mu c}$ is the characteristics of Eyring-Powell model, $\frac{\partial P}{\partial X}=$ constant, $\mathrm{U}$ implies dimensionless velocity, $\mathrm{t}$ stands for dimensionless time, $\mathrm{T}$ represents dimensionless temperature function, $\mathrm{N}$ stands for thermal radiation, Pr represents Prandtl number, Da is Darcy number, Re signifies Reynold number, Gr implies thermal Grashof number, $M$ implies Magnetic field parameter, Ec stands for Eckert number and $\lambda$ is the wall-slip parameter.

\section{NUMERICAL SOLUTION}

The finite difference equations relating to equations (11), (15) and (6) respectively are

$R e \frac{U_{j}^{k+1}-U_{j}^{k}}{\Delta t}=\eta\left[\frac{U_{j-1}^{k+1}-2 U_{j}^{k+1}+U_{j+1}^{k+1}+U_{j-1}^{k}-2 U_{j}^{k}+U_{j+1}^{k}}{2(\Delta Y)^{2}}\right]+$
$G_{r}\left(\frac{T_{j}^{k+1}+T_{j}^{K}}{2}\right)-\left(M^{2}+D a\right)\left(\frac{U_{j}^{k+1}+U_{j}^{k}}{2}\right)$

$R e \frac{U_{j}^{k+1}-U_{j}^{k}}{\Delta t}=[1+A]\left[\frac{U_{j-1}^{k+1}-2 U_{j}^{k+1}+U_{j+1}^{k+1}+U_{j-1}^{k}-2 U_{j}^{k}+U_{j+1}^{k}}{2(\Delta Y)^{2}}\right]+$

$G_{r}\left(\frac{T_{j}^{k+1}+T_{j}^{K}}{2}\right)-\left(M^{2}+D a\right)\left(\frac{U_{j}^{k+1}+U_{j}^{k}}{2}\right)$

$R e \frac{T_{j}^{k+1}-T_{j}^{k}}{\Delta t}=\frac{1}{p r}\left[\frac{T_{j-1}^{k+1}-2 T_{j}^{k+1}+T_{j+1}^{k+1}+T_{j-1}^{k}-2 T_{j}^{k}+T_{j+1}^{k}}{2(\Delta Y)^{2}}\right]-$

$\frac{N}{\operatorname{Pr}}\left[\frac{T_{j}^{k+1}+T_{j}^{k}}{2}\right]+\frac{E c\left(U_{j+1}^{k}-U_{j-1}^{k}\right)^{2}}{4(\Delta Y)^{2}}$

With these underlying conditions

$U_{j}^{k+1}=0, u_{j}^{k}=0, T_{j}^{k+1}=0, T_{j}^{k}=0, \forall j, t=0$

$U_{j-1}^{k+1}=\frac{-2 \Delta y U_{j}^{k+1}}{\lambda}+U_{j+1}^{k+1}, T_{j-1}^{k+1}=0, j=1, t>0$

$U_{j-1}^{k}=\frac{-2 \Delta y U_{j}^{k}}{\lambda}+U_{j+1}^{k}, T_{j-1}^{k}=0, j=1, t>0$

$U_{j}^{k+1}=0, T_{j}^{k+1}=1, U_{j}^{k}=0, T_{j}^{k}=1, j=n, t>0$

The velocity and temperature at $Y=0$ respectively are $U_{0}$ and $T_{0}$ while $U_{n}$ and $T_{n}$ are respectively the corresponding velocity and temperature at $Y=1$ with the interval $\Delta Y=\frac{1}{n}$.

In the calculations, subscript $j$ and superscript $k$ assign grid points along $Y$ and $t$ directions respectively. The estimations of $U$ and $T$ at $t=0$ are known at all grid points from the underlying conditions. The calculation of $U$ and $T$ for the next time level $(k+1)$ th utilizing the known esteems at past time level are assessed by framing tri-diagonal system of equations from the finite difference equation (16), (17) and (18). The estimations of $T$ at each nodal point at any step length was resolved utilizing the known esteems at past time. Thomas algorithm was utilized to explain this tri-diagonal arrangement of equationss. Accordingly, the estimations of $T$ are computed at each nodal point on a particular $j$ at $(k+1)$ th time level. Utilizing the estimations of $T$ at $(k+1)$ th time level in Eq. (17), the estimations of $U$ at $(k+1)$ th time level are computed also in the same manner. Subsequently the estimations of $T$ and $U$ are known on a specific $j$-level for Eyring-Powell model. Similarly, making use of the values of $T$ at $(k+1)$ th time level in Eq. (16), the values of $U$ at $(k+1)$ th time level are ascertained which gives the estimations of $T$ and $U$ on a specific $j$-level for PrandtlEyring model.

\section{RESULTS AND DISCUSSION}

Numerical investigation was carried out to analyze the flow of Prandtl-Eyring and Eyring-Powell models on the velocity and temperature profiles.

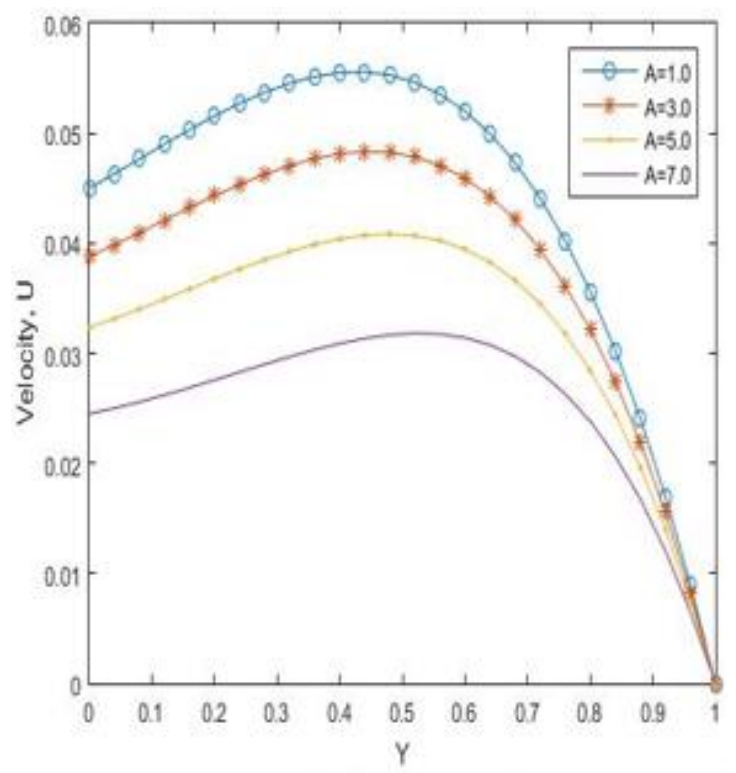

Figure 1. Velocity distribution for different values of EyringPowel parameter (A)

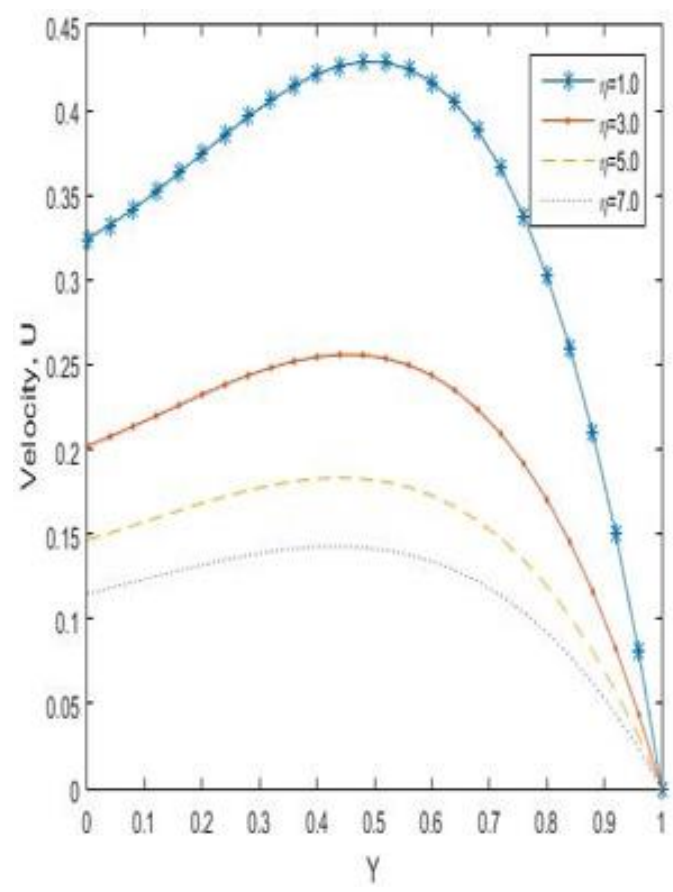

Figure 2. Velocity distribution for different values of Prandtl-Eyring parameter $(\eta)$

Figures 1 and 2 delineates the velocity distributions for various values of non-Newtonian parameters $\mathrm{A}$ and $\eta$. The Prandtl-Eyring and Eyring-Powell fluids velocity are decreasing functions of fluid parameters $\mathrm{A}$ and $\eta$. It was seen that increasing both parameters $\mathrm{A}$ and $\eta$ causes diminishment 
in the rate of flow as observed on their velocity distributions. This retarding effect is due to the presence of Lorentz force within the fluid layers. The velocity variation in PrandtlEyring fluid is higher than that of Eyring-Powell fluid. This diminishment represents the low rate of flow of NonNewtonian fluids.

Figures 3 and 4 represents temperature distributions for various values of non-Newtonian parameters $\mathrm{A}$ and $\eta$. Increasing both $\mathrm{A}$ and $\eta$ causes the temperature distribution to increase. This shows that rapid increase in the temperature with $\mathrm{A}$ and $\eta$ are guaranteed at the wall.

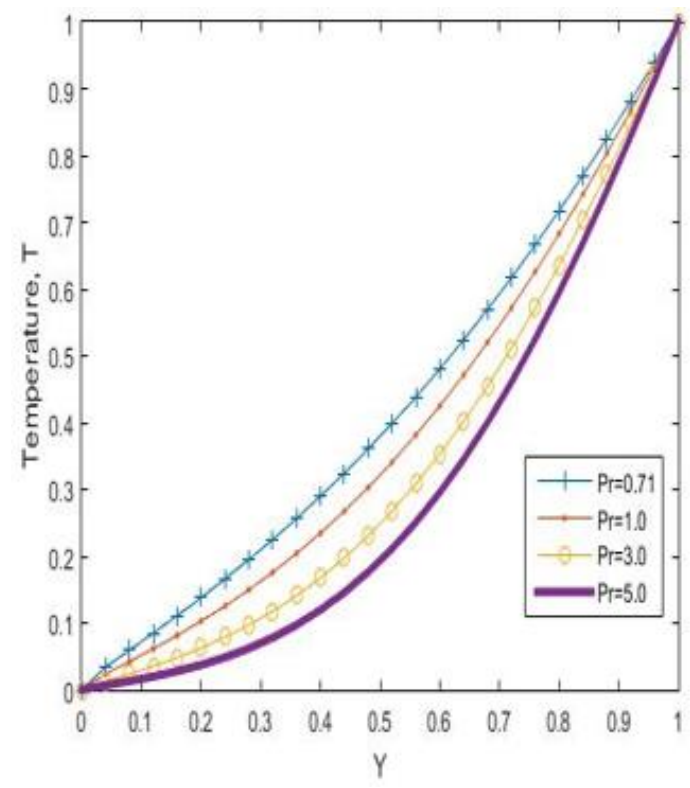

Figure 3. Temperature distribution for different values of Prandtl number (Pr) for Eyring-Powel fluid

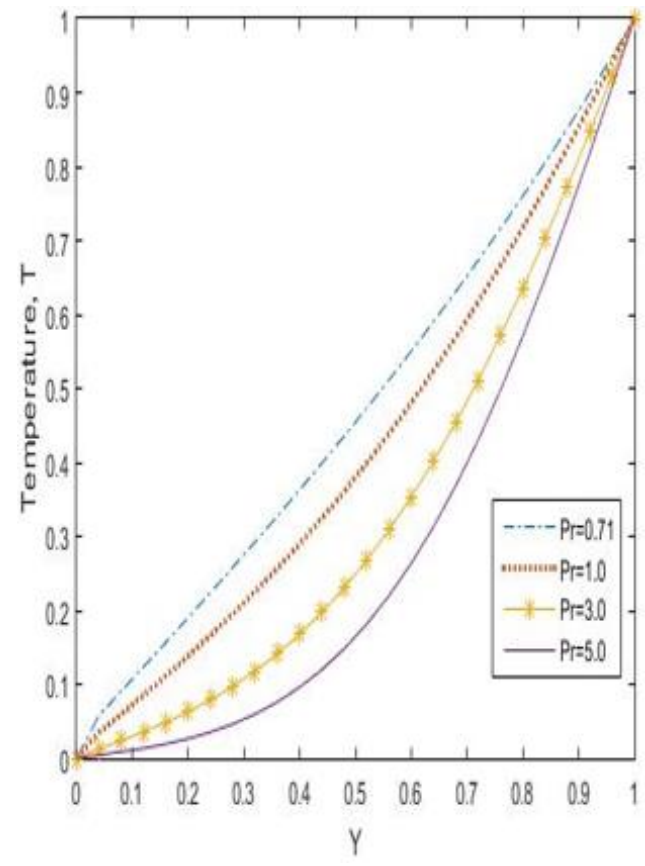

Figure 4. Temperature distribution for different values of Prandtl number (Pr) for Prandtl-Eyring fluid

The effects of Prandtl number on velocity distributions for Prandtl-Eyring and Eyring-Powell models is reported on figures 5 and 6 respectively. Prandtl number portrays the proportion of thicknesses of the viscous and thermal boundary layers. Increasing Prandtl number causes the velocity profiles to decrease.

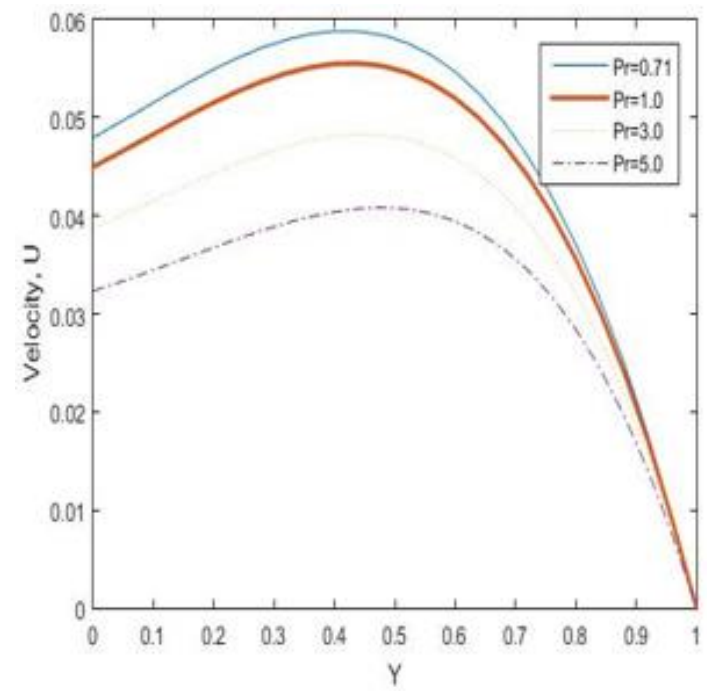

Figure 5. Velocity distribution for different values of Prandtl number (Pr) for Eyring-Powel fluid

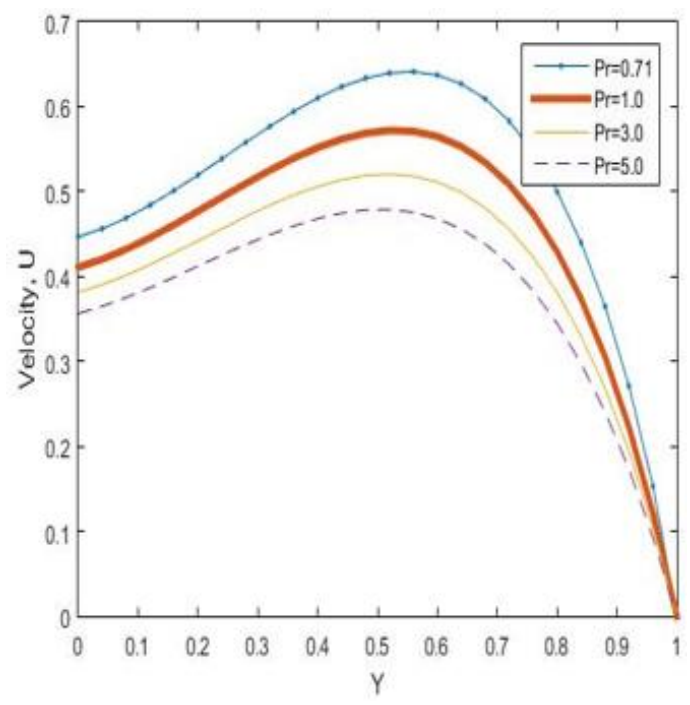

Figure 6. Velocity distribution for different values of Prandtl number $(\operatorname{Pr})$ for Prandtl-Eyring fluid

\section{CONCLUSION}

The numerical examination for an unsteady MHD nonNewtonian fluid in a permeable medium is considered. NonNewtonian fluid models considered are Prandtl Eyring and Eyring Powell liquid. The resulting equations from the governing equation after non dimensionalising form coupled nonlinear partial differential equations which is solved using the implicit finite difference scheme of Crank Nicolson. It is noticed that,

The velocity distribution in Prandtl-Eyring fluid is quite higher than the Eyring Powell fluid.

The temperature variation for $\operatorname{Pr}=0.71,1.0 .3 .0$ and 5.0 in Eyring-Powell fluid is a little slower than that of PrandtlEyring fluid.

Considering the importance of Non-Newtonian fluid models, other models can also be taken into account 


\section{REFERENCES}

[1] Timol MG, Kalthia NL. (1985). Group theoretic approach to similarity solutions in non-Newtonian natural convection flows. Journal of Energy Heat and Mass Transfer 7(4): 251-288.

[2] Eldaebe NTM, Hassan AA, Mona AA. (2003). Effect of couple stresses on the MHD of a non-Newtonian unsteady flow between two parallel porous plates. Journal of physics 58: 204-210. http://dx.doi.org/10.1515/zna-2003-0405

[3] Zueco J, Beg OA. (2009). Network numerical simulation applied to pulsatile non-Newtonian flow through a channel with couple stress and wall mass effects. International Journal of Applied Mathematics and Mech. 5: $1-16$.

[4] Ara A, Khan NA, Khan H, Sultan F. (2014). Radiation effects on boundary layer flow of an Eyring-Powell fluid over an exindent potentially shrinking sheet. Ain Shams Engineering Journal 5: 1337-1342.

[5] Malik MY, Hussian A, Nadeems. (2013). Boundary layer flow of an Eyring-Powell model fluid due to a stretching cylinder with variable viscosity. Journal of Scientia Iranica 20(2): 313-321. https://doi.org/10.1016/j.scient.2013.02.028

[6] Hayat T, Asad S, Mustafa M, Alsaedi A. (2014). Radiation effects on the flow of Powell Eyring fluid past an unsteady inclined stretching sheet with non-uniform Heat Source/Sink. PLOS ONE 9(7): e103214. https://doi:10.1371/journal. pone. 0103214

[7] Khader MM, Megahed AM. (2013). Numerical studies for flow and heat transfer of the Powell-Eyring fluid thin film over an unsteady stretching sheet with internal heat generation using the finite difference method. Journal of Applied Mechanics Technical Phys. 5(4): 440-450. https://doi.org/10.1134/S0021894413030139

[8] Arifuzzaman SM, Khan MS, Hossain KE, Islam MS, Akter S, Roy R. (2017). Chemically reactive viscoelastic fluid flow in presence of nano particle through porous stretching sheet. Frontiers in Heat and Mass Transfer 9(5): 1-11. http://dx.doi.org/10.5098/hmt.9.5

[9] Khan MS, Karim I, Ali LE, Islam A. (2012). Unsteady MHD free convection boundary-layer flow of a nanofluid along a stretching sheet with thermal radiation and viscous dissipation effects. International Nano Letters 2(24). https://doi.org/10.1186/2228-5326-2-24

[10] Arifuzzaman SM, Rana BMJ, Ahmed R, Ahmmed SF. (2017). Cross diffusion and MHD effect on a high order chemically reactive micropolar fluid of naturally convective heat and mass transfer past through an infinite vertical porous medium with a constant heat sink. AIP Conference Proceedings 1851, 020006. http://dx.doi.org/10.1063/1.4984635

[11] Arifuzzaman SM, Khan MS, Islam MS, Islam MM, Rana BMJ, Biswas P, Ahmmed SF. (2017). MHD Maxwell fluid flow in presence of nano-particle through a vertical porous-plate with heat- generation, radiation absorption and chemical reaction. Frontiers in Heat and Mass Transfer 9(25): 1-14. http://dx.doi.org/10.5098/hmt.9.25

[12] Zaman H. (2013). unsteady incompressible couette flow problem for the Eyring-Powell model with porous walls. American Journal of Computational Mathematics 3: 313 325. http://doi 10.4236/ajcm.2013.34041
[13] Gbadeyan JA, Dada MS. (2013). On the influence of radiation radiation and heat transfer on an unsteady MHD Non-Newtonian fluid flow with slip in a porous medium. Journal of Mathematical Research 5(3): 40-49. http://dx.doi.org/10.5539/jmr.v5n3p40

[14] Adesanya SO, Gbadeyan JA. (2011). Adomia Decomposition approach to steady visco-elastic flow with slip through a planar channel. Journal of nonlinear science 11(1): 86-94.

[15] Mahmoudi A, Mejri I. (2015). Analysis of conductionradiation heat transfer with variable thermal conductivity and variable refractive index: Application of the Lattice Boltzmann method. International Journal of Heat and $\begin{array}{lll}\text { Technology } & 33(1): & 1-8 \text {. }\end{array}$ https://doi.org/10.18280/ijht.330101

[16] Darji RM, Timol MG. (2013). Group-theoretic similarity analysis for natural convection boundary layer flow of a class of non-Newtonian fluids. International Journal of Advanced Scientific and Technical Research 3(1): 54-69.

\section{NOMENCLATURE}

g gravitational acceleration

$\mathrm{k}$ permeability

$\mathrm{u} \quad$ Velocity in the $\mathrm{x}$ direction

$\mathrm{v} \quad$ Velocity in the $\mathrm{y}$ direction

$\mathrm{t}^{*} \quad$ Dimensional time

t Dimensionless time

$x^{*}, y^{*}$ Dimensional coordinates along the plate and normal to the plate

$\mathrm{X}$ Dimensionless coordinate along the plate

Y Dimensionless coordinate normal to the plate

h Characteristic length

$\mathrm{N}$ Thermal radiation parameter

$\mathrm{P}^{*} \quad$ Dimensional pressure

P Dimensionless fluid pressure

$\mathrm{T}^{*} \quad$ Dimensional temperature of fluid

$\mathrm{T}$ Dimensionless temperature of fluid

A Eyring-Powell parameter

$B_{0} \quad$ Applied magnetic field

$C_{p} \quad$ specific heat at constant pressure

$\mathrm{D}_{\mathrm{a}}$ Darcy number

$\mathrm{E}_{\mathrm{c}} \quad$ Eckert number

$\mathrm{G}_{\mathrm{r}}$ Thermal Grashof number

$P_{r} \quad$ Prandtl number

$\mathrm{q}_{\mathrm{r}} \quad$ radiative heat flux

$\mathrm{R}_{\mathrm{e}} \quad$ Reynolds number

$\mathrm{T}_{\mathrm{w}} \quad$ Temperature of the plate

\section{Greek alphabets}

Viscosity

$\rho$ density

Stefan-Boltzmann constant

$\alpha, c \quad$ characteristic of Eyring-Powell model

$\beta$ and $b$ characteristic of Prandtl-Eyring model

$\eta \quad$ Prandtl-Eyring parameter

$\tau_{x y} \quad$ stress tenso 